\title{
LIMIT DISTRIBUTIONS OF POLYNOMIAL TRAJECTORIES ON HOMOGENEOUS SPACES
}

\author{
NIMISH A. SHAH
}

1. Introduction. Let $G$ be a Lie group and $\Gamma$ a lattice in $G$; that is, $\Gamma$ is a discrete subgroup of $G$ such that $G / \Gamma$ admits a finite $G$-invariant measure. Let $u: \mathbf{R} \rightarrow G$ be a unipotent one-parameter subgroup of $G$; that is, $\operatorname{Ad} u(t)$ is a unipotent linear automorphism of $\operatorname{Lie}(G)$ for all $t \in \mathbf{R}$. The action of $\{u(t): t \in \mathbf{R}\}$ on $G / \Gamma$ is called a unipotent flow.

Through the series of four fundamental papers [R1], [R2], [R3], [R4] proving the Raghunathan conjectures on "nice algebraic" behaviour of unipotent flows, Marina Ratner proved also the following result: For any $x \in G / \Gamma$, there exists a closed subgroup $F$ of $G$ such that the orbit $F x$ is closed and admits a unique $F$-invariant probability measure, say $\mu_{F}$, and the trajectory $\{u(t) x: t>0\}$ is uniformly distributed with respect to $\mu_{F}$. That is, for any bounded continuous function $f$ on $G / \Gamma$,

$$
\lim _{T \rightarrow \infty} \frac{1}{T} \int_{0}^{T} f(u(t) x) d t=\int_{F x} f d \mu_{F} .
$$

Essentially the basic property of a unipotent one-parameter subgroup used in the work of Ratner is that the map $t \mapsto \operatorname{Ad} u(t)$ is a polynomial function in each coordinate of $\operatorname{End}(\operatorname{Lie}(G))$. Therefore it is natural to ask the following question. Let $G$ be a closed subgroup of $\operatorname{SL}_{n}(\mathbf{R})$, and $\Gamma$ a lattice in $G$. Let $\theta: \mathbf{R} \rightarrow G$ be a map which is a polynomial function, namely, each matrix coordinate is a polynomial. Then is it true that the trajectory $\{\theta(t) \Gamma: t>0\}$ is uniformly distributed with respect to a measure of the form $\mu_{F}$ as above? In the case when $G=\mathbf{R}^{n}$ and $\Gamma=\mathbf{Z}^{n}$, this indeed holds, as can be deduced from a classical result due to Weyl. In this paper we answer the question affirmatively in a more general setup.

A group $G$ is called real algebraic if it is an open subgroup of $\mathbf{R}$-points of an algebraic group $\mathbf{G}$ defined over $\mathbf{R}$. A map $\Phi: \mathbf{R}^{k} \rightarrow G$ is called regular algebraic if it is the restriction of a morphism $\Phi: \mathbf{C}^{k} \rightarrow \mathbf{G}$ of algebraic varieties defined over $\mathbf{R}$. We caution the reader that a map such as $\phi: \mathbf{R} \rightarrow \mathbf{R}^{*}$ given by $\phi(t)=1+t^{2}$ for all $t \in \mathbf{R}$ is not regular algebraic according to our definition, as $\phi$ does not extend to an algebraic map from $\mathbf{C}$ to $\mathbf{C}^{*}$.

The following is the main result.

Received 3 November 1993.

Author's research at MSRI supported by NSF grant DMS 8505550 . 
THEOREM 1.1. Let $G$ be a real algebraic group, and let $\Delta \subset G_{1} \subset G$ be closed subgroups such that $G_{1} / \Delta$ admits a finite $G_{1}$-invariant measure. Let $k \in \mathbf{N}$ and $\Theta$ : $\mathbf{R}^{k} \rightarrow G$ be a map defined as $\Theta\left(t_{1}, \ldots, t_{k}\right)=\theta_{k}\left(t_{k}\right) \cdots \theta_{1}\left(t_{1}\right)$ for all $\left(t_{1}, \ldots, t_{k}\right) \in \mathbf{R}^{k}$, where $\theta_{i}: \mathbf{R} \rightarrow G$ is a regular algebraic map for $i=1, \ldots, k$. Suppose that $\Theta(0)=e$ and $\Theta\left(\mathbf{R}^{k}\right) \subset G_{1}$. Then there exists a closed subgroup $F$ of $G$ containing $\Theta\left(\mathbf{R}^{k}\right)$ such that the orbit $F \Delta$ is closed in $G / \Delta$ and admits a unique $F$-invariant probability measure, say $\mu_{F}$, and the following holds: given sequences $T_{n}^{(1)} \rightarrow \infty, \ldots, T_{n}^{(k)} \rightarrow \infty$ as $n \rightarrow \infty$, for the boxes $B_{n}=\left[0, T_{n}^{(1)}\right] \times \cdots \times\left[0, T_{n}^{(k)}\right]$ we have

$$
\lim _{n \rightarrow \infty} \frac{1}{m\left(B_{n}\right)} \int_{\mathbf{t} \in B_{n}} f(\Theta(t) \Delta) d m(\mathbf{t})=\int_{F \Delta} f d \mu_{F},
$$

for all $f \in \mathrm{C}_{\mathrm{c}}(G / \Delta)$, the space of continuous functions with compact support on $G / \Delta$, $m$ being the Lebesgue measure on $\mathbf{R}^{k}$.

Our proof crucially involves Ratner's theorem [R3] on the measure rigidity of unipotent flows. It also requires the technique of relating the action of $G$ on thin neighbourhoods in $G / \Delta$ of images of compact subsets of certain algebraic subvarieties of $G$, with linear actions of $G$ on thin neighbourhoods of compact subsets of certain algebraic subvarieties in finite-dimensional vector spaces. This linearisation procedure was first used in [DS] and later developed further in [DM2], [S], and [DM3].

We will deduce the following fact as a consequence of our proof of Theorem 1.1 .

Corollary 1.1. Let $G, \Delta$, and $G_{1}$ be as in Theorem 1.1. Let $\theta: \mathbf{R} \rightarrow G$ be a regular algebraic map such that $\theta(\mathbf{R}) \subset G_{1}$ and $\theta(0)=e$. Then there exists a closed subgroup $F$ of $G$ containing $\theta(\mathbf{R})$ such that the orbit $F \Delta$ is closed and admits a unique $F$-invariant probability measure, say $\mu_{F}$, and the following holds: for any $k \geqslant 1$ and $f \in \mathrm{C}_{\mathrm{c}}(G / \Delta)$ we have

$$
\lim _{T \rightarrow \infty} \frac{1}{T} \int_{0}^{T} f\left(\theta\left(t^{1 / k}\right) \Delta\right) d t=\int_{F \Delta} f d \mu_{F} .
$$

The main theorem is proved only for the product type of regular algebraic functions $\Theta$. Using the above corollary, we obtain a version of it which holds for all regular algebraic functions.

Corollary 1.2. Let $G, \Delta$, and $G_{1}$ be as in Theorem 1.1. Let $\Theta: \mathbf{R}^{k} \rightarrow G$ be a regular algebraic map such that $\Theta(0)=e$ and $\Theta\left(\mathbf{R}^{k}\right) \subset G_{1}$. Then there exists a closed subgroup $F$ of $G$ containing $\Theta\left(\mathbf{R}^{k}\right)$ such that the orbit $F \Delta$ is closed and admits a unique $F$-invariant probability measure, say $\mu_{F}$, and the following holds: for any $f \in \mathrm{C}_{\mathrm{c}}(G / \Delta)$,

$$
\lim _{R \rightarrow \infty} \frac{1}{m\left(B_{R}\right)} \int_{\mathbf{t} \in B_{R}} f(\Theta(\mathbf{t}) \Delta) d m(\mathbf{t})=\int_{F \Delta} f d \mu_{F},
$$

where $B_{R}$ denotes the ball of radius $R$ in $\mathbf{R}^{k}$ centered at the origin. 
It may be emphasized that in this result for all regular algebraic maps $\Theta$, the averaging is allowed only on balls, rather than on the boxes as in Theorem 1.1 whose sizes could increase at different rates in different coordinates.

Using the results we answer affirmatively a question raised by Ratner in [R4], [R5] regarding the limit distributions of orbits of higher-dimensional unipotent subgroups on finite-volume homogeneous spaces of Lie groups. First we recall some notation from [R1].

Let $N$ be a connected simply connected nilpotent group with Lie algebra $\underline{\mathbf{n}}$. Let $B=\left\{b_{1}, \ldots, b_{k}\right\}$ be a basis in $\underline{\mathbf{n}}$. For $v \in \underline{\mathbf{n}}$, write $v=\sum_{i=1}^{k} \alpha_{i}(v) b_{i}$. We say that the basis $B$ is triangular if $\alpha_{k}\left(\left[b_{i}, b_{j}\right]\right)=0$ for all $k \leqslant \max \{i, j\}$ and all $i, j=1, \ldots, k$. Any permutation of a triangular basis is called a regular basis.

COROLlaRY 1.3. Let $G$ be a Lie group, $\Gamma$ a closed subgroup of $G$ such that $G / \Gamma$ admits a finite $G$-invariant measure, and $N$ a simply connected unipotent subgroup of $G$. Let $\left\{b_{1}, \ldots, b_{k}\right\}$ be a regular basis in $\underline{\mathbf{n}}$. For $s_{1}, \ldots, s_{k}>0$ define

$$
\mathscr{S}\left(s_{1}, \ldots, s_{k}\right)=\left\{\left(\exp t_{k} b_{k}\right) \cdots\left(\exp t_{1} b_{1}\right) \in N: 0 \leqslant t_{j} \leqslant s_{j}, j=1, \ldots, k\right\} .
$$

Then for any $x \in G / \Gamma$, there exists a closed subgroup $F$ of $G$ containing $N$ such that the orbit $F x$ is closed and admits a unique $F$-invariant probability measure, say $\mu_{F}$, and the following holds: for any $f \in \mathrm{C}_{\mathrm{c}}(G / \Gamma)$,

$$
\lim _{s_{1} \rightarrow \infty, \ldots, s_{k} \rightarrow \infty} \frac{1}{\lambda\left(\mathscr{S}\left(s_{1}, \ldots, s_{k}\right)\right)} \int_{h \in \mathscr{S}\left(s_{1}, \ldots, s_{k}\right)} f(h x) d \lambda(h)=\int_{F x} f d \mu_{F},
$$

where $\lambda$ denotes a Haar measure on $N$.

In Section 2 we make some reductions so that the results proved elsewhere for a homogeneous space $G / \Gamma$, where $\Gamma$ is a lattice, can be applied to the homogeneous space $G_{1} / \Delta$. In Section 3 we modify a result of Dani and Margulis about returning of any unipotent trajectory to compact sets with large densities on a finite-volume homogeneous space, in order to obtain the same result for polynomial trajectories. In Section 4 we show that any limiting distribution of a polynomial trajectory is invariant under a nontrivial unipotent one-parameter subgroup and apply the measure rigidity of unipotent flows due to Ratner. In Section 5 we develop further the method of linearising $G$-action on certain subsets of $G / \Delta$ as mentioned earlier in the introduction. In the last section we complete the proofs of the results stated above.

We conclude this introduction with a natural question. Let $\theta$ be a map as in the Corollary 1.1. Then does there exist a closed subgroup $F$ of $G$ containing $\theta(Z)$ such that the orbit $F \Delta$ is closed and admits a unique $F$-invariant probability measure, say $\mu_{F}$, and for any $f \in \mathrm{C}_{\mathrm{c}}(G / \Delta)$,

$$
\lim _{N \rightarrow \infty} \frac{1}{N} \sum_{n=0}^{N-1} f(\theta(n) \Delta)=\int_{F \Delta} f d \mu_{F} ?
$$


In the case when $G$ is a nilpotent group, this question can be answered affirmatively (cf. [CFS, Chapter 7], for the abelian case). But even in the simplest semisimple case of $G=G_{1}=\mathrm{SL}_{2}(\mathbf{R}), \Delta=\mathrm{SL}_{2}(Z)$, and the regular algebraic map $\theta(t)=\left(\begin{array}{cc}1 & t^{2} \\ 0 & 1\end{array}\right)$ for all $t \in \mathbf{R}$, the question is unanswered.

Thanks are due to G. A. Margulis for suggestions regarding the formulation of the main theorem and help in resolving some technical difficulties in the proof. The author wishes to thank S. G. Dani, Alex Eskin, Shahar Mozes, and Marina Ratner for several useful discussions. This work was mainly carried out while the author was visiting the Mathematical Sciences Research Institute at Berkeley, California.

2. Some reductions. We begin by noting the following.

Proposition 2.1. Let $G$ be a real algebraic group, and $\Theta: \mathbf{R}^{k} \rightarrow G$ a regular algebraic map such that $\Theta(0)=e$.

(1) Let $L$ be the smallest closed subgroup of $G$ containing $\Theta\left(\mathbf{R}^{k}\right)$. Then $L$ is generated by algebraic unipotent one-parameter subgroups of $G$.

(2) Suppose further that there exist closed subgroups $\Delta \subset G_{1} \subset G$ such that $G_{1} / \Delta$ admits a finite $G_{1}$-invariant measure and $\Theta\left(\mathbf{R}^{k}\right) \subset G_{1}$. Let $F$ be the smallest closed subgroup containing $\Theta\left(\mathbf{R}^{k}\right)$ such that the orbit $F \Delta$ is closed. Then $F /(F \cap \Delta)$ admits a finite $F$-invariant measure, and the Zariski closure of $F \cap \Delta$ contains $F$.

Proof. Let $\tilde{L}$ denote the Zariski closure of $L$ in $G$. Recall that any regular algebraic map from $\mathbf{R}^{k}$ to $\mathbf{R}^{*}$ or to a compact algebraic group is constant. Therefore, by the definition of $L$, we have that $\tilde{L}$ has no nontrivial toral or compact factors. Hence $\tilde{L}$ is generated by algebraic unipotent one-parameter subgroups of $G$. In particular, the radical of $L$ is unipotent, and hence $L=\tilde{L}$. This proves (1).

Let $\tilde{\Delta}$ denote the Zariski closure of $\Delta$ in $G$. By a version of Borel's density theorem as in [D2, Theorem 4.1], all unipotent one-parameter subgroups of $G_{1}$ are contained in $\tilde{\Delta}$, in particular $L \subset \tilde{\Delta}$. Now, replacing $G_{1}$ by $G_{1} \cap \tilde{\Delta}$ and $G$ by $\tilde{\Delta}$, we can assume that $\Delta$ is Zariski dense in $G$ and, in particular, $\Delta^{0}$, the connected component of $e$ in $\Delta$, is a normal subgroup of $G$.

Now let $\phi: G \rightarrow G / \Delta^{0}$ denote the quotient homomorphism. Then $\phi(\Delta)$ is a discrete subgroup of $\phi(G)$. And the map $\bar{\phi}: G / \Delta \rightarrow \phi(G) / \phi(\Delta)$, defined as $\bar{\phi}(g \Delta)=$ $\phi(g) \phi(\Delta)$ for all $g \in G$, is an equivariant isomorphism. Now $\phi(F)$ is the smallest closed subgroup of $\phi\left(G_{1}\right)$ containing $\phi(L)$ such that $\phi(F) \phi(\Delta)$ is closed. By [S, Theorem 2.3], $F /(F \cap \Delta) \cong \phi(F) /(\phi(F) \cap \phi(\Delta))$ admits a finite $F$-invariant measure.

Now, by the above Borel density argument, $L$ is contained in the Zariski closure of $F \cap \Delta$. Therefore, due to the definition of $F$, the Zariski closure of $F \cap \Delta$ also contains $F$. This proves (2).

Note 2.1. In view of Proposition 2.1, by replacing $\Delta$ by $F \cap \Delta, G_{1}$ by $F$, and $G$ by the Zariski closure of $F$, we may assume that there is no proper algebraic subgroup $A$ of $G$ such that $\Theta\left(\mathbf{R}^{k}\right) \subset A$ and $A \Delta$ is closed. 
Note 2.2. Let $W$ be the closed subgroup generated by all algebraic unipotent one-parameter subgroups of $G$ contained in $\Delta^{0}$. Then $W$ is a normal subgroup of $G$. Let $q: G \rightarrow G / W$ be the natural quotient map. By [S, Lemma 2.9], $W$ is a real algebraic group. Therefore $G / W$ is a real algebraic group and $q$ is an algebraic morphism. Note that $G / \Delta \cong q(G) / q(\Delta)$ equivariantly. Therefore without loss of generality we can replace $\Delta$ by $q(\Delta), G_{1}$ by $q\left(G_{1}\right), G$ by $q(G)$, and $\Theta$ by $q \circ \Theta$. In view of this we can assume that $\Delta$ contains no nontrivial algebraic unipotent one-parameter subgroups of $G$.

Note 2.3. Also, without loss of generality we may assume that $\theta_{k}$ is nonconstant.

3. Relative measures of a large compact set in $G / \Delta$ on polynomial trajectories. We need a modified version of an important result due to Dani and Margulis on the asymptotic behaviour of unipotent trajectories on finite-volume homogeneous spaces of Lie groups. First we recall some elementary facts and fix some notation.

LEMMA 3.1. Let $E$ and $F$ be Borel measurable subsets of a bounded interval $I \subset[0, \infty)$, and let $k \geqslant 1$. Suppose there exist nonnegative reals $\varepsilon_{1}<1 / k$ and $\varepsilon_{2}<$ $1 / k$ such that

$$
\ell(E) \leqslant \varepsilon_{1} \cdot \ell(I) \quad \text { and } \quad \ell(F) \geqslant\left(1-\varepsilon_{2}\right) \cdot \ell(I),
$$

where $\ell$ denotes the Lebesgue measure on $\mathbf{R}$. Then

$$
\ell\left(E^{k}\right) \leqslant\left(k \varepsilon_{1}\right)\left(1-k \varepsilon_{2}\right)^{-1} \cdot \ell\left(F^{k}\right),
$$

where $E^{k}:=\left\{s^{k}: s \in E\right\}$.

Proof. Let $I=[a, b]$. Then

$$
\begin{aligned}
\ell\left(E^{k}\right) & =\int_{a^{k}}^{b^{k}} \chi_{E^{k}}(t) d t \\
& =k \cdot \int_{a}^{b} \chi_{E}(s) s^{k-1} d s \\
& \leqslant k \cdot \ell(E) b^{k-1} \\
& \leqslant k \cdot \ell(E)\left(b^{k}-a^{k}\right) /(b-a) \\
& \leqslant\left(k \varepsilon_{1}\right) \cdot \ell\left(I^{k}\right) .
\end{aligned}
$$

Similarly, we have

$$
\ell\left(F^{k}\right)=\ell\left(I^{k}\right)-\ell\left((I \backslash F)^{k}\right) \geqslant\left(1-k \varepsilon_{2}\right) \cdot \ell\left(I^{k}\right) .
$$

The result follows from equations (1) and (2). 
LEMMA 3.2. Let $B$ be a bounded open convex subset of $\mathbf{R}^{k}$. Let $S$ be the unit sphere in $\mathbf{R}^{k}$ centered at the origin. Fix $\mathbf{t}_{0} \in B$. For any subset $E$ of $B$, and any $\mathbf{x} \in S$, define

$$
E_{\mathbf{x}}=\left\{t>0: t \mathbf{x}+t_{0} \in E\right\}
$$

Let $E$ and $F$ be measurable subsets of $B$. Suppose there are: a subset $D \subset B$, containing $E$ and $F$, and nonnegative reals $\varepsilon_{1} \leqslant 1 / k$ and $\varepsilon_{2} \leqslant 1 / k$ such that, for every $\mathbf{x} \in S$, the set $D_{\mathbf{x}}$ is open in $[0, \infty)$ and, for every connected component I of $D_{\mathbf{x}}$, we have

$$
\ell\left(E_{\mathbf{x}} \cap I\right) \leqslant \varepsilon_{1} \cdot \ell(I) \quad \text { and } \quad \ell\left(F_{\mathbf{x}} \cap I\right) \geqslant\left(1-\varepsilon_{2}\right) \cdot \ell(I)
$$

Then

$$
m(E) \leqslant\left(k \varepsilon_{1}\right)\left(1-k \varepsilon_{2}\right)^{-1} \cdot m(F)
$$

where $m$ denotes the Lebesgue measure on $\mathbf{R}^{k}$.

Proof. Let $\sigma$ denote the rotation-invariant probability measure on $S$ such that the volume of a unit ball in $\mathbf{R}^{k}$ is $\sigma(S) / k$. Using polar decomposition of $B$ at the pole $\mathbf{t}_{0}$, we have

$$
\begin{aligned}
m(E) & =\int_{\mathbf{x} \in S} d \sigma(\mathbf{x}) \cdot \int_{0}^{\ell\left(B_{\mathbf{x}}\right)} \chi_{E_{\mathbf{x}}}(t) t^{k-1} d t \\
& =(1 / k) \int_{\mathbf{x} \in S} d \sigma(\mathbf{x}) \cdot \int_{0}^{\ell\left(\boldsymbol{B}_{\mathbf{x}}\right)^{k}} \chi_{E_{\mathbf{x}}}\left(t^{1 / k}\right) d t \\
& =(1 / k) \int_{\mathbf{x} \in S} \ell\left(E_{\mathbf{x}}^{k}\right) d \sigma(\mathbf{x}) \\
& \leqslant\left(k \varepsilon_{1}\right)\left(1-k \varepsilon_{2}\right)^{-1} \cdot(1 / k) \int_{\mathbf{x} \in S} \ell\left(F_{\mathbf{x}}^{k}\right) d \sigma(\mathbf{x}) \\
& =\left(k \varepsilon_{1}\right)\left(1-k \varepsilon_{2}\right)^{-1} \cdot m(F),
\end{aligned}
$$

where the inequality follows from Lemma 3.1. This completes the proof.

Notation. Let $G$ be a Lie group. For $k, l \in \mathbf{N}$, let $\mathscr{P}_{l}\left(\mathbf{R}^{k}, G\right)$ denote the set of continuous maps $\Theta: \mathbf{R}^{k} \rightarrow G$ such that, for all $\mathbf{c}, \mathbf{a} \in \mathbf{R}^{k}$ and $X \in \operatorname{Lie}(G)$, the map

$$
t \in \mathbf{R} \mapsto \operatorname{Ad} \circ \Theta(t \mathbf{c}+\mathbf{a})(X) \in \operatorname{Lie}(G)
$$

is a polynomial of degree at most $l$ in each coordinate of $\operatorname{Lie}(G)$. 
Let $\mathscr{P}_{l}(G)$ denote the set $\mathscr{P}_{l}\left(\mathbf{R}^{1}, G\right)$. Note that if $\theta \in \mathscr{P}_{l}(G)$ is a group homomorphism, then $\theta$ is a one-parameter Ad-unipotent subgroup of $G$, and the converse holds for $l=\operatorname{dim} G-1$.

THEOREM 3.1. Let $G$ be a Lie group and $\Delta$ a closed subgroup such that $G / \Delta$ admits a finite $G$-invariant measure. Then, given a compact set $C \subset G / \Delta$, an $\varepsilon>0$, and an $l \in \mathbf{N}$, there exists a compact subset $K \subset G / \Delta$ with the following property: for any $x \in G / \Delta$, and $\Theta \in \mathscr{P}_{l}\left(\mathbf{R}^{k}, G\right)$, and any bounded open convex set $B \subset \mathbf{R}^{k}$, if $\Theta(B) x \cap C \neq \varnothing$, then

$$
\frac{1}{m(B)} \cdot m(\{\mathbf{t} \in B: \Theta(\mathbf{t}) x \in K\})>(1-\varepsilon) .
$$

Proof. In [DM3, Theorem 6.1], the result is stated for an Ad-unipotent oneparameter subgroup $u: \mathbf{R} \rightarrow G$, in the place of $\Theta$ as above. The proof uses only the property that $u \in \mathscr{P}_{d}(G)$, where $d=\operatorname{dim} G-1$, rather than the fact that $\{u(t)\}$ is also a one-parameter subgroup. Hence essentially the same proof applies for all $\theta \in \mathscr{P}_{l}(G)$.

Now choose a compact set $K$ such that the conclusion of the theorem is valid for $\varepsilon / k$ in place of $\varepsilon$ and $\theta \in \mathscr{P}_{l}(G)$ in place of $\Theta$.

Let $B$ be as in the hypothesis and take $\mathbf{t}_{0} \in B$ such that $\Theta\left(\mathbf{t}_{0}\right) x \in C$. Define

$$
E=\{\mathbf{t} \in B: \Theta(\mathbf{t}) x \notin K\} .
$$

Let $S$ denote the unit sphere in $\mathbf{R}^{k}$ centered at the origin. Fix $\mathbf{x} \in S$. Define a map $\theta(t)=\Theta\left(t \mathbf{x}+\mathbf{t}_{0}\right)$ for all $t \in \mathbf{R}$. Then $\theta \in \mathscr{P}_{l}(G)$. Therefore

$$
\ell\left(E_{\mathbf{x}}\right)=\ell\left(\left\{t \in B_{\mathbf{x}}: \theta(t) x \notin K\right\}\right)<(\varepsilon / k) \cdot \ell\left(B_{\mathbf{x}}\right) .
$$

Therefore, by Lemma 3.2 , for $F=D=B, \varepsilon_{1}=\varepsilon / k$, and $\varepsilon_{2}=0$, we get

$$
m(E) \leqslant \varepsilon \cdot m(B)
$$

This completes the proof.

Limit distributions of a polynomial trajectory on $G / \Delta$. Let $G, G_{1}, \Delta$, and $\Theta$ be as in Theorem 1.1. Let $\mathscr{P}(G / \Delta)$ denote the space of Borel probability measures on $G / \Delta$ with the weak topology. For any bounded open convex set $B \subset \mathbf{R}^{k}$, let $\mu_{B} \in \mathscr{P}(G / \Delta)$ be such that, for any $f \in \mathrm{C}_{\mathrm{c}}(G / \Delta)$,

$$
\int_{G / \Delta} f d \mu_{B}=\frac{1}{m(B)} \int_{\mathbf{t} \in B} f(\Theta(\mathbf{t}) \Delta) d \mathbf{t} .
$$

CoROllary 3.1. Given a sequence $\left\{B_{n}\right\}_{n \in \mathbf{N}}$ of bounded open convex subsets of $\mathbf{R}^{k}$ containing 0 , there exist a strictly increasing sequence $\left\{n_{i}\right\}_{i \in \mathbf{N}} \subset \mathbf{N}$ and a measure $\mu \in \mathscr{P}(G / \Delta)$ such that $\mu_{B_{n_{i}}} \rightarrow \mu$ as $i \rightarrow \infty$. 
Proof. Using the existence of limits in the space of probability measures on the one-point compactification of $G / \Delta$, say $X^{*}$, we obtain a subsequence $\left\{\mu_{n_{i}}\right\}$ converging to a probability measure $\mu$ on $X^{*}$. Since $\Theta\left(\mathbf{R}^{k}\right) \Delta \subset G_{1} / \Delta$, due to Theorem 3.1, for any $\varepsilon>0$ there exists a compact set $K \subset G / \Delta$ such that $\mu_{B_{n}}(K) \geqslant$ $1-\varepsilon$ for all $n \in \mathbf{N}$. Therefore $\mu(K) \geqslant 1-\varepsilon$, and hence $\mu(G / \Delta)=1$.

Note 3.1. In view of Corollary 3.1, to prove Theorem 1.1, it is enough to show the following: For $i=1, \ldots, k$, let sequences $T_{n}^{(i)} \rightarrow \infty$ as $n \rightarrow \infty$ be given. Put $B_{n}=\left[0, T_{n}^{(1)}\right] \times \cdots \times\left[0, T_{n}^{(k)}\right]$ for all $n \in \mathbf{N}$. Suppose that $\mu_{B_{n}} \rightarrow \mu$ in $\mathscr{P}(G / \Delta)$ as $n \rightarrow \infty$. Let $F$ be the smallest closed subgroup of $G$ containing $\Delta^{0}$ and $\Theta\left(\mathbf{R}^{k}\right)$ such that the orbit $F \Delta$ is closed. Then $\mu$ is $F$-invariant and $\mu(F \Delta)=1$.

4. Invariance under a unipotent flow. In this section we show that the limiting distribution $\mu$ as in Note 3.1 is invariant under the action of the nontrivial unipotent one-parameter subgroup of $G$. This result allows us to apply Ratner's measure rigidity theorem in our study.

Using the following observation, we associate nontrivial unipotent elements to nonconstant regular algebraic maps into algebraic groups (cf. [DM1, Proposition 2.4]).

LEMMA 4.1. Let $G$ be a real algebraic group and $\theta: \mathbf{R} \rightarrow G$ a nonconstant regular algebraic map. Then there exists $a q \geqslant 0$ and $a$ nontrivial algebraic unipotent one-parameter subgroup $\rho: \mathbf{R} \rightarrow G$ such that, for any $s \in \mathbf{R}$,

$$
\lim _{t \rightarrow \infty} \theta\left(t+s t^{-q}\right) \theta(t)^{-1}=\rho(s)
$$

Proof. Let $M(n, \mathbf{R})$ denote the affine space of $n \times n$ real matrices which contains $G$ as an affine subvariety. There exist polynomials $\theta_{i j}(t)$ for $i, j=1, \ldots, n$, such that $\theta(t)=\left(\theta_{i j}(t)\right)_{n \times n}$. Put

$$
d=\operatorname{deg}(\theta(t)):=\max _{i, j=1, \ldots, n} \operatorname{deg}\left(\theta_{i j}(t)\right)
$$

For $\xi \in \mathbf{R}$, we have

$$
\theta(t+\xi)=\theta(t)+\sum_{l=1}^{d} \theta^{(l)}(t) \frac{\xi^{l}}{l !}
$$

Note that the $l$ th derivative $\theta^{(l)}(t)$ is a regular algebraic map of degree $d-l$. Since the map $g \rightarrow g^{-1}$ is an algebraic morphism on $G$, we have that the map $t \mapsto \theta(t)^{-1}$ is also regular algebraic. Put

$$
q=\max _{1 \leqslant l \leqslant d}(1 / l) \operatorname{deg}\left(\theta^{(l)}(t) \theta(t)^{-1}\right) \geqslant 0
$$


Then for every $1 \leqslant l \leqslant d$,

$$
\lim _{t \rightarrow \infty} \theta^{(l)}(t) \theta(t)^{-1} t^{-q l}=\lambda_{l} \in M(n, \mathbf{R}),
$$

and $\left(\lambda_{1}, \ldots, \lambda_{d}\right) \neq 0$. Put

$$
\rho(s)=I+\sum_{l=1}^{d} \lambda_{l} \frac{s^{l}}{l !}
$$

for all $s \in \mathbf{R}$. Then, for any $s \in \mathbf{R}$ and any map $t \mapsto s_{t}$ with $s_{t} \rightarrow s$ as $t \rightarrow \infty$, we have

$$
\lim _{t \rightarrow \infty} \theta\left(t+s_{t} t^{-q}\right) \theta(t)^{-1}=\rho(s) .
$$

Now, for $s_{1}, s_{2} \in \mathbf{R}$,

$$
\begin{aligned}
\rho\left(s_{1}+s_{2}\right) \rho\left(s_{2}\right)^{-1}= & \lim _{t \rightarrow \infty}\left(\theta\left(t+\left(s_{1}+s_{2}\right) t^{-q}\right) \theta(t)^{-1}\right) \cdot\left(\theta(t) \theta\left(t+s_{2} t^{-q}\right)^{-1}\right) \\
= & \lim _{t \rightarrow \infty} \theta\left(y_{t}+s_{t} y_{t}^{-q}\right) \theta\left(y_{t}\right)^{-1} \\
& \text { where } y_{t}=t+s_{2} t^{-q} \text { and } s_{t}=s_{1}\left(y_{t} / t\right)^{q} \\
= & \rho\left(s_{1}\right) .
\end{aligned}
$$

Thus, by equations (3), (4), and (5), $\rho: \mathbf{R} \rightarrow G$ is a nontrivial algebraic group homomorphism. Therefore $\rho$ is a nontrivial algebraic unipotent one-parameter subgroup of $G$. This completes the proof.

We digress to modify the above result in the next lemma for its use in proving Corollary 1.1 later.

LemMA 4.2. Let the notation be as in Lemma 4.1. Take $k \geqslant 1$. Put $\psi(t)=\theta\left(t^{1 / k}\right)$ for all $t>0$. Then for $q_{1}=(1 / k)(q+1)-1>-1$ and every $s \in \mathbf{R}$,

$$
\lim _{t \rightarrow \infty} \psi\left(t+s t^{-q_{1}}\right) \psi(t)^{-1}=\rho(s / k),
$$

where $\rho$ is the unipotent one-parameter subgroup as in the conclusion of Lemma 4.1.

Proof. Using Taylor's expansion, we get

$$
\left(t+s t^{-q_{1}}\right)^{1 / k}=t^{1 / k}+s_{t} t^{-q_{1}-1+1 / k}=y_{t}+s_{t} y_{t}^{-k\left(q_{1}+1-1 / k\right)},
$$

where $y_{t}=t^{1 / k}$, and $s_{t} \rightarrow s / k$ as $t \rightarrow \infty$. Now the result follows from equation (4). 
We need the following elementary fact.

LEMMA 4.3. For any bounded continuous function $f$ on $\mathbf{R}$, any $q>-1$, and $s \in \mathbf{R}$,

$$
\lim _{T \rightarrow \infty} \frac{1}{T} \int_{1}^{T} f\left(t+s t^{-q}\right)-f(t) d t=0
$$

where the rate of convergence depends only on $s, q$, and $\sup |f|$, rather than $f$ itself.

The next result is the first main step in the proof of Theorem 1.1.

Proposition 4.1. Let the measure $\mu$ be as constructed in Note 3.1. Then $\mu$ is invariant under a nontrivial unipotent one-parameter subgroup of $G$.

Proof. Due to Note 2.3, $\theta_{k}$ is nonconstant. Obtain $q>-1$ and a nontrivial unipotent one-parameter subgroup $\rho: \mathbf{R} \rightarrow G$ as in Lemma 4.1 for $\theta_{k}$ in place of $\theta$. To show that $\mu$ is invariant under the action of $\rho$, take any $s \in \mathbf{R}$ and any $f \in$ $\mathrm{C}_{\mathrm{c}}(G / \Delta)$. Then

$$
\begin{aligned}
\int_{G / \Delta} f(\rho(s) x) d \mu(x)= & \lim _{n \rightarrow \infty} \frac{1}{m\left(B_{n}\right)} \int_{\mathbf{t}=\left(t_{1}, \ldots, t_{k}\right) \in B_{n}} f\left(\rho(s) \theta\left(t_{k}\right) \cdots \theta\left(t_{1}\right) \Delta\right) d m(\mathbf{t}) \\
= & \lim _{n \rightarrow \infty} \frac{1}{m\left(B_{n}\right)} \int_{t=\left(t_{1}, \ldots, t_{k-1}\right) \in\left[0, T_{n}^{(1)}\right] \times \cdots \times\left[0, T_{n}^{(k-1)}\right]} d t_{1} \cdots d t_{k-1} \\
& \cdot\left(\int_{t_{k} \in\left[1, T^{(k)}\right]} f\left(\theta_{k}\left(t_{k}+s t_{k}^{-q}\right) \theta_{k-1}\left(t_{k-1}\right) \cdots \theta_{1}\left(t_{1}\right) \Delta\right) d t_{k}\right) \\
= & \lim _{n \rightarrow \infty} \frac{1}{m\left(B_{n}\right)} \int_{t \in B_{n}} f\left(\Theta\left(t_{1}, \ldots, t_{k}\right) \Delta\right) d \mathbf{t} \\
= & \int_{G / \Delta} f(x) d \mu(x),
\end{aligned}
$$

where the second equality follows from the choice of $\rho$ and the uniform continuity of $f$, and the third equality follows from Lemma 4.3 applied to the integration in the variable $t_{k}$. This completes the proof.

Description of measures invariant under a unipotent flow. To describe a finite invariant measure of unipotent flow using Ratner's classification of finite ergodic invariant measures, we introduce some notation. We note that the results of this subsection and of Section 5 hold under a weaker assumption that $G$ is any Lie group and $\Delta$ is a closed subgroup of $G$ such that $\Delta^{0}$ is normal in $G$. 
Let $\mathscr{H}$ be the collection of all closed connected subgroups $H$ of $G$ such that (1) $H \supset \Delta^{0}$, (2) $H / H \cap \Delta$ admits a finite $H$-invariant measure, and (3) the subgroup generated by all unipotent one-parameter subgroups of $H$ acts ergodically on $H / H \cap \Delta$ with respect to the $H$-invariant probability measure. Note that by Proposition 2.1, the Zariski closure of $\operatorname{Ad}(H \cap \Delta)$ contains $\operatorname{Ad}(H)$.

THEOREM 4.1 (Ratner). The collection $\mathscr{H}$ is countable.

Proof. See [R3, Theorem 1.1] or [DM3, Proposition 2.1] for different proofs of the result.

Let $W$ be a subgroup of $G$ which is generated by one-parameter unipotent subgroups of $G$ contained in $W$. For any $H \in \mathscr{H}$, define

$$
\begin{aligned}
& N(H, W)=\left\{g \in G: W \subset g H g^{-1}\right\}, \\
& S(H, W)=\bigcup_{\substack{H^{\prime} \in \mathcal{H} \\
\operatorname{dim} H^{\prime}<\operatorname{dim} H}} N\left(H^{\prime}, W\right) .
\end{aligned}
$$

Let $\pi: G \rightarrow G / \Delta$ denote the natural quotient map. Note that (see [MS, Lemma 2.4])

$$
\pi(N(H, W) \backslash S(H, W))=\pi(N(H, W)) \backslash \pi(S(H, W)) .
$$

The following version of Ratner's measure ridigity theorem was obtained in [MS, Theorem 2.2].

THEOREM 4.2 (Ratner). Let $W$ be a subgroup as above and $\mu \in \mathscr{P}(G / \Delta)$ a $W$-invariant measure. For every $H \in \mathscr{H}$, let $\mu_{H}$ denote the restriction of $\mu$ on $\pi(N(H, W) \backslash S(H, W))$. Then the following hold.

1. For all Borel-measurable subsets $A \subset G / \Delta$,

$$
\mu(A)=\sum_{H \in \mathscr{H} *} \mu_{H}(A)
$$

where $\mathscr{H}^{*} \subset \mathscr{H}$ is a countable set consisting of one representative from each $\Delta$-conjugacy class of elements in $\mathscr{H}$.

2. Each $\mu_{H}$ is $W$-invariant. For any $W$-ergodic component $v \in \mathscr{P}(G / \Delta)$ of $\mu_{H}$, there exists a $g \in N(H, W)$ such that $v$ is the (unique) $g \mathrm{Hg}^{-1}$-invariant probability measure on the closed orbit $g H \Delta$.

To understand the measure $\mu$ as in Proposition 4.1, we should study the action of $G$ in thin neighbourhoods of images in $G / \Delta$ of compact subsets of $N(H, W) \backslash$ $S(H, W)$. In the following section we show that, on these thin neighbourhoods, the $G$-action is equivariant with the linear $G$-action on thin neighbourhoods of compact subsets of an affine algebraic subvariety in a finite-dimensional linear $G$-space. In the next section we carry out this linearisation process. 
5. Linearisation of $G$-action on thin neighbourhoods of $\pi(N(H, W) \backslash S(H, W))$. Let $H \in \mathscr{H}$. Let $\mathbf{g}$ denote the Lie algebra of $G$ and $\underline{\mathbf{h}}$ its subalgebra associated to $H$. For $d=\operatorname{dim} \underline{h}$, put $V_{H}=\bigwedge^{d} \underline{g}$, the $d$ th exterior power, and consider the linear $G$-action on $V_{H}$ via the representation $\wedge^{d}$ Ad, the $d$ th exterior of the adjoint representation of $G$ on g. Fix $p_{H} \in \bigwedge^{d} \underline{h} \backslash\{0\}$, and let $\eta_{H}: G \rightarrow V_{H}$ be the map defined by $\eta_{H}(g)=g p_{H}=\left(\bigwedge^{d} \mathrm{Ad} g\right) p_{H}$ for all $g \in G$. Note that

$$
\eta_{H}^{-1}\left(p_{H}\right)=\left\{g \in N(H): \operatorname{det}\left(\left.\operatorname{Ad} g\right|_{\underline{\underline{h}}}\right)=1\right\}
$$

where $N(H)$ denotes the normalizer of $H$ in $G$; we denote this subgroup by $N^{1}(H)$.

Put $\Delta_{H}=N(H) \cap \Delta$. For any $\delta \in \Delta_{H}$, we have $\delta(H \Delta / \Delta)=H \Delta / \Delta$, and hence $\delta$ preserves the volume of $H \Delta / \Delta$. Therefore $\left|\operatorname{det}\left(\left.\operatorname{Ad} \delta\right|_{\underline{h}}\right)\right|=1$, and hence $\delta p_{H}= \pm p_{H}$.

In view of this, we define $\bar{V}_{H}=V_{H} /\{\mathrm{Id},-\mathrm{Id}\}$ if $\Delta_{H}^{-} p_{H}=\left\{p_{H},-p_{H}\right\}$, and $\bar{V}_{H}=V_{H}$ if $\Delta_{H} p_{H}=p_{H}$. The action of $G$ factors through the quotient map from $V_{H}$ onto $\bar{V}_{H}$. Let $\bar{p}_{H}$ denote the image of $p_{H}$ in $\bar{V}_{H}$, and define $\bar{\eta}_{H}: G \rightarrow \bar{V}_{H}$ as $\bar{\eta}_{H}(g)=g \bar{p}_{H}$ for all $g \in G$. Then $\Delta_{H}=\bar{\eta}_{H}^{-1}\left(\bar{p}_{H}\right) \cap \Delta$.

For any subset $Z$ of $G / \Delta$, define

$$
\operatorname{Rep}(Z):=\left\{g \bar{p}_{H} \in \bar{V}_{H}: g \in G, \pi(g) \in Z\right\}
$$

THEOREM 5.1 [DM3, Theorem 3.4]. The orbit $\Delta \cdot \bar{p}_{H}$ is closed, and hence discrete. In particular, the following hold.

(1) The orbit $N^{1}(H) \Delta$ is closed in $G / \Delta$.

(2) For every $x \in G / \Delta$, the set $\operatorname{Rep}(x)$ is discrete in $\bar{V}_{H}$.

(3) For any compact set $Z \subset G / \Delta$, the set $\operatorname{Rep}(Z)$ is closed in $\bar{V}_{H}$.

(4) The map $\phi: G / \Delta_{H} \rightarrow G / \Delta \times \bar{V}_{H}$ defined by

$$
\phi\left(g \Delta_{H}\right)=\left(\pi(g), \bar{\eta}_{H}(g)\right) \quad(\forall g \in G)
$$

is proper.

Let $W$ be a subgroup of $G$ which is generated by unipotent one-parameter subgroups of $G$ contained in $W$.

Proposition 5.1 [DM3, Proposition 3.2]. Let $A_{H}$ denote the Zariski closure of $\bar{\eta}_{H}(N(H, W))$ in $\bar{V}_{H}$. Then

$$
\bar{\eta}_{H}^{-1}\left(A_{H}\right)=N(H, W)
$$

Proposition 5.2. Let $H \in \mathscr{H}$ and $D$ be a compact subset of $A_{H}$. Let $K$ be a compact subset of $G / \Delta$. Define

$$
\mathscr{S}(D, K)=\left\{g \in G: \pi(g) \in K, g \bar{p}_{H} \in D, \text { and } g \delta \bar{p}_{H} \in D \text { for some } \delta \in \Delta \backslash \Delta_{H}\right\} .
$$


Then the following hold.

(1) $\pi(\mathscr{S}(D, K))$ is compact.

(2) There exist $m \in \mathbf{N}$ and $F_{i} \in \mathscr{H}$ with $F_{i} \subset H$ and $\operatorname{dim} F_{i}<\operatorname{dim} H$ for $1 \leqslant i \leqslant$ $m$ such that

$$
\mathscr{S}(D, K) \subset \bigcup_{i=1}^{m} N\left(F_{i}, W\right) \Delta .
$$

(3) Given any compact set $K_{1} \subset K \backslash \pi(\mathscr{S}(D, K))$, there exists a neighbourhood $\Phi$ of $D$ in $\bar{V}_{H}$ such that, for any $x \in K_{1}$, the set $\operatorname{Rep}(x) \cap \Phi$ is either empty or a singleton set.

Proof. Statements (1) and (2) follow from [DM3, Proposition 7.2]. Statement (3) follows from [DM3, Corollary 3.5].

Dynamics of polynomial trajectories in thin neighbourhoods. The following growth property of polynomial maps has turned out to be of great significance in the study of polynomial trajectories near affine algebraic varieties.

Proposition 5.3 [DM3, Proposition 4.2]. Let a compact set $C \subset A_{H}$, an $\varepsilon>$ 0 , and an $l \in \mathbf{N}$ be given. Then there exists a larger compact set $D \subset A_{H}$ such that, for any neighbourhood $\Phi$ of $D$ in $\bar{V}_{H}$ the following property holds: there exists a neighbourhood $\Psi$ of $C$ in $\bar{V}_{H}$ with $\Psi \subset \Phi$ such that, for any $\theta \in \mathscr{P}_{l}(G)$, any element $w \in \bar{V}_{H}$, and any bounded interval $(a, b)$ of $\mathbf{R}$, if $\theta(a) w \notin \Phi$, then

$$
\ell(\{t \in(a, b): \theta(t) w \in \Psi\})<\varepsilon \cdot \ell(\{t \in(a, b): \theta(t) w \in \Phi\}) .
$$

Proof. This result is proved in [DM3, Proposition 4.2] for a unipotent oneparameter subgroup $u: \mathbf{R} \rightarrow G$ in place of $\theta$. The fact that $u \in \mathscr{P}_{d}(G)$ for $d=$ $\operatorname{dim} G-1$ is the only property of $u$ which is used in the proof. The same proof goes through for any $\theta \in \mathscr{P}_{l}(G)$.

We need the following result for our proof of Theorem 1.1. This result and the other related results are also of independent interest. For rest of the section we further assume that $G / \Delta$ admits a finite $G$-invariant measure.

THEOREM 5.2. Given a compact set $C_{1} \subset N(H, W) \backslash S(H, W)$, an $\varepsilon>0$, and an $l \in \mathbf{N}$, there exists a neighbourhood $\Omega$ of $\pi\left(C_{1}\right)$ in $G / \Delta$ such that, for any $x \in G / \Delta$ and any $\Theta \in \mathscr{P}_{l}\left(\mathbf{R}^{k}, G\right)$, one of the following conditions is satisfied.

(i) There exists $g \in G$ such that

$$
\Theta\left(\mathbf{R}^{k}\right) x \subset g N^{1}(H) \Delta .
$$

(Recall that due to Theorem 5.1 the orbit $g N^{1}(H) \Delta$ is closed.)

(ii) There exists a bounded open convex set $B^{\prime} \subset \mathbf{R}^{k}$ such that, for all bounded open convex subsets $B$ of $\mathbf{R}^{k}$ containing $B^{\prime}$,

$$
\frac{1}{m(B)} m(\{\mathbf{t} \in B: \Theta(\mathbf{t}) x \in \Omega\})<\varepsilon .
$$


For $k=1$, the result follows from [MS, Proposition 3.4]. The case of $k>1$ is much more involved. We give a proof by induction on $\operatorname{dim} H$. For this purpose we need to prove a more technical version of it.

Proposition 5.4. Let $\varepsilon>0, l \in \mathbf{N}$, and a compact set $K \subset G / \Delta$ be given. Let $C_{1}$ be a compact subset of $N(H, W)$. Then there exist compact sets $D \subset A_{H}$ and $S_{1} \subset$ $\bigcup_{i=1}^{m} N\left(F_{i}, W\right)$, where $m \in \mathbf{N}$, and $F_{i} \in \mathscr{H}$ with $F_{i} \subset H$ and $\operatorname{dim} F_{i}<\operatorname{dim} H$ for $1 \leqslant$ $i \leqslant m$, such that the following holds: Let a neighbourhood $\Phi$ of $D$ in $\bar{V}_{H}$ and a compact set $Z \subset K \backslash \pi\left(S_{1}\right)$ be given. Then there exists a neighbourhood $\Omega$ of $\pi\left(C_{1}\right)$ in $G / \Delta$ such that, for any $x \in G / \Delta, \Theta \in \mathscr{P}_{l}\left(\mathbf{R}^{k}, G\right)$, and a bounded open convex set $B \subset \mathbf{R}^{k}$, one of the following conditions is satisfied:

(1) $\Theta(B) x \cap Z=\varnothing$.

(2) $\Theta(B) v \subset \Phi$ for some $v \in \operatorname{Rep}(x)$.

(3)

$$
\frac{1}{m(B)} m(\{\mathbf{t} \in B: \Theta(\mathbf{t}) x \in \Omega\})<\varepsilon
$$

For $k=1$ the result can be concluded from the proof of [DM3, Theorem 7.3]. The general case requires a different type of arrangement in the proof. First we derive Theorem 5.2 from Proposition 5.4.

Proof of Theorem 5.2. Let $K$ be the closure of a relatively compact neighbourhood of $\pi\left(C_{1}\right)$. Obtain $D$ and $S_{1}$ using Proposition 5.4.

Due to equation (6), $\pi\left(C_{1}\right) \cap \pi(S(H, W))=\varnothing$. Therefore there exists a neighbourhood of $\pi\left(C_{1}\right)$ contained in $K$ with its closure $Z$ such that $Z \cap \pi\left(S_{1} \cup\right.$ $\mathscr{S}(D, K))=\varnothing$. Using Proposition 5.2, obtain a neighbourhood $\Phi$ of $D$ in $\bar{V}_{H}$ such that every $x \in Z$ has at most one representative in $\Phi$. Now using Proposition 5.4, obtain a neighbourhood $\Omega$ of $\pi\left(C_{1}\right)$ contained in $Z$ such that at least one of the three possibilities of its conclusion holds.

Suppose there exists a bounded open convex set $B_{0} \subset \mathbf{R}^{k}$ for which the possibility (3) fails to hold. Since $\Omega \subset Z$, the possibility (1) cannot hold. Therefore the possibility (2) must hold.

Then there exists $\mathbf{t}_{0} \in \mathbf{R}^{k}$ and $v \in \operatorname{Rep}(x)$ such that $\Theta\left(\mathbf{t}_{0}\right) x \in Z$ and $\Theta\left(\mathbf{t}_{0}\right) v \in \Phi$. Such a $v$ is unique due to the choice of $\Phi$. Since $\mathbf{t} \mapsto \Theta(\mathbf{t}) v$ is an algebraic morphism from $\mathbf{R}^{k}$ to $\bar{V}_{H}$, either (a) $\Theta\left(\mathbf{R}^{k}\right) v=\Theta\left(\mathbf{t}_{0}\right) v$, or (b) there exists $\mathbf{t}_{1} \in \mathbf{R}^{k}$ such that $\Theta\left(\mathbf{t}_{1}\right) v \notin \Phi$.

First suppose (a) holds. Then there exists $g_{1} \in \pi^{-1}(x)$ such that $v=g_{1} \bar{p}_{H}$, and since the stabilizer of $v$ in $G$ is $g_{1} N^{1}(H) g_{1}^{-1}$, we have $\Theta\left(\mathbf{R}^{k}\right) g_{1} \subset g N^{1}(H)$, where $g=\Theta\left(\mathbf{t}_{0}\right) g_{1}$. Thus the possibility (i) of the present theorem holds.

Now suppose (b) holds. Then, for any open convex set $B \supset B_{0} \cup\left\{t_{1}\right\}$, the possibilities (1) or (2) cannot hold, and hence the possibility (3) must hold. Thus possibility (ii) of the present theorem holds. This completes the proof.

For the purpose of proving Proposition 5.4 by using induction on $\operatorname{dim} H$, we need its following consequence. 
Corollary 5.1. Let $\varepsilon>0, l \in \mathbf{N}$, and a compact set $K \subset G / \Delta$ be given. Let $C_{1}$ be a compact subset of $\bigcup_{i=1}^{n} N\left(H_{i}, W\right)$, where $n \in \mathbf{N}$ and $H_{i} \in \mathscr{H}$ for $1 \leqslant i \leqslant n$. Then there exists a compact set $D_{1} \subset \bigcup_{i=1}^{n} N\left(H_{i}, W\right)$ such that the following holds: given a compact set $Z \subset K \backslash \pi\left(D_{1}\right)$, there exists a neighbourhood $\Omega$ of $\pi\left(C_{1}\right)$ in $G / \Delta$ such that, for any $x \in G / \Delta, \Theta \in \mathscr{P}_{l}\left(\mathbf{R}^{k}, G\right)$, and a bounded open convex set $B \subset \mathbf{R}^{k}$, either

$$
\Theta(B) x \cap Z=\varnothing
$$

or

$$
\frac{1}{m(B)} m(\{\mathbf{t} \in B: \Theta(\mathbf{t}) x \in \Omega\})<\varepsilon
$$

Proof. We prove the result for $n=1$; the general case follows easily from this. Using Proposition 5.4 for $H=H_{1}$, obtain compact sets $D \subset A_{H}$ and

$$
S_{1} \subset \bigcup_{i=1}^{m} N\left(F_{i}, W\right)
$$

where $m \in \mathbf{N}$, where $F_{i} \in \mathscr{H}$ with $F_{i} \subset H$, and where $\operatorname{dim} F_{i}<\operatorname{dim} H$ for $1 \leqslant i \leqslant k$.

By Theorem 5.1(4) and Proposition 5.1, there exists a compact set $\widetilde{D} \subset$ $N(H, W)$ such that

$$
\{x \in K: \operatorname{Rep}(x) \in D\}=\pi(\tilde{D})
$$

Now $D_{1}=S_{1} \cup \tilde{D}$ is a compact subset $N(H, W)$.

Let $Z \subset K \backslash \pi\left(D_{1}\right)$ be a given compact set. Then $\operatorname{Rep}(Z) \cap D=\varnothing$. By Theorem $5.1, \operatorname{Rep}(Z)$ is closed in $\bar{V}_{H}$. Therefore there exists a neighbourhood $\Phi$ of $D$ in $\bar{V}_{H}$ such that

$$
\Phi \cap \operatorname{Rep}(Z)=\varnothing
$$

Suppose that $\Theta(B) x \cap Z \neq \varnothing$. Then the possibilities (1) and (2) of Proposition 5.4 cannot hold, due to equation (9). Therefore the possibility (3) of the proposition must hold, and hence equation (8) holds. This completes the proof.

Proof of Proposition 5.4. Let $\Omega_{1}$ be a relatively compact neighbourhood of $\pi\left(C_{1}\right)$ in $G / \Delta$. By Theorem 3.1, there exists a compact set $K_{1} \subset G / \Delta$ such that, for any $y \in \overline{\Omega_{1}}, \theta \in \mathscr{P}_{l}(G)$, and $T>0$,

$$
\frac{1}{T} \ell\left(\left\{t \in[0, T]: \theta(t) x \in K_{1}\right\}\right)>1-1 /(4 k) .
$$


For the compact set $C=C_{1} \bar{p}_{H} \subset A_{H}$, obtain a compact set $D \subset A_{H}$ such that the conclusion of Proposition 5.3 is satisfied for $\varepsilon /(4 k)$ in place of $\varepsilon$.

As we mentioned earlier, we shall prove this theorem by induction on $\operatorname{dim} H$. Note that when $\operatorname{dim} H$ is small, $S(H, W)=\varnothing$ and hence $\mathscr{S}\left(D, K_{1}\right)=\varnothing$. We begin with some observations for which we first assume that $\mathscr{S}\left(D, K_{1}\right) \neq \varnothing$.

By Proposition 5.2, there exists $m \in \mathbf{N}$ and, for each $1 \leqslant i \leqslant m$, there exists $F_{i} \in \mathscr{H}$ with $F_{i} \subset H$ and $\operatorname{dim} F_{i}<\operatorname{dim} H$ such that

$$
\mathscr{S}\left(D, K_{1}\right) \subset \bigcup_{i=1}^{m} N\left(F_{i}, W\right) \Delta .
$$

Also there exists a compact set $C_{2} \subset \bigcup_{i=1}^{m} N\left(F_{i} W\right)$ such that

$$
\pi\left(C_{2}\right)=\pi\left(\mathscr{S}\left(D, K_{1}\right)\right)
$$

By induction, we can assume that the present proposition is true for each $F_{i}$. Hence Corollary 5.1 is valid for $C_{2}$ in place of $C_{1}$ and $F_{i}$ in place of $H_{i}$ in its statement. Thus we obtain a compact set

$$
S_{2} \subset \bigcup_{i=1}^{m} N\left(F_{i}, W\right)
$$

such that the following holds: given any compact set $Z_{1} \subset \overline{\Omega_{1}} \backslash \pi\left(S_{2}\right)$, there exists a neighbourhood $\Omega_{2}$ of $\pi\left(\mathscr{S}\left(D, K_{1}\right)\right)$ such that, for any $y \in Z_{1}, \theta \in \mathscr{P}_{l}(G)$, and $T>0$,

$$
\frac{1}{T} \ell\left(\left\{t \in[0, T]: \theta(t) y \in \Omega_{2}\right\}\right)<1 /(4 k)
$$

Again we apply Corollary 5.1 as above for $S_{2}$ in place of $C_{1}$ and obtain a compact set

$$
S_{1} \subset \bigcup_{i=1}^{m} N\left(F_{i}, W\right)
$$

such that the following holds: given a compact set $Z \subset K \backslash \pi\left(S_{1}\right)$ as in the statement of the proposition, there exists a neighbourhood $\Omega_{3}$ of $\pi\left(S_{2}\right)$ such that, for the given $x \in G / \Delta, \Theta \in \mathscr{P}_{l}\left(\mathbf{R}^{k}, G\right)$, and the bounded open convex set $B \subset \mathbf{R}^{k}$, either

$$
\Theta(B) x \cap Z=\varnothing
$$

or

$$
\frac{1}{m(B)} m\left(\left\{\mathbf{t} \in B: \Theta(\mathbf{t}) x \in \Omega_{3}\right\}\right)<\varepsilon /(4 k)
$$


Put

$$
Z_{1}=\overline{\Omega_{1}} \backslash \Omega_{3}
$$

and obtain a neighbourhood $\Omega_{2}$ of $\pi\left(\mathscr{S}\left(D, K_{1}\right)\right)$ such that equation (11) holds. Suppose, if $\mathscr{S}\left(D, K_{1}\right)=\varnothing$, the above equations are satisfied if we put $S_{2}=S_{1}=$ $\varnothing$ and $\Omega_{3}=\Omega_{2}=\varnothing$.

Let $\Phi$ be a given neighbourhood of $D$ as in the statement of the present proposition. Using Proposition 5.2, we replace $\Phi$ by a smaller neighbourhood such that every $y \in K_{1} \backslash \Omega_{2}$ has at most one representative in $\Phi$.

By the choice of $D$, there exists a neighbourhood $\Psi$ of $C$ in $\bar{V}_{H}$ with $\Psi \subset \Phi$ such that, for any $\theta \in \mathscr{P}_{l}(G)$, any $v \in \bar{V}_{H}$, and an interval $(a, b)$, if $\theta(a) v \notin \Phi$, then

$$
\ell(\{t \in[a, b]: \theta(t) v \in \Psi\})<\varepsilon /(4 k) \cdot \ell(\{t \in[a, b]: \theta(t) v \in \Phi\}) .
$$

Put

$$
\Omega=\left\{y \in \Omega_{1}: \operatorname{Rep}(y) \cap \Psi \neq \varnothing\right\}
$$

Then $\Omega$ is an open neighbourhood of $\pi\left(C_{1}\right)$.

After having made the above constructions, we start analysing possibilities (1), (2), and (3) of the conclusion of the proposition. First suppose that possibility (2) does not hold. Take any $v \in \operatorname{Rep}(x)$. Then there exists $\mathbf{t}_{v} \in B$ such that $\Theta\left(\mathbf{t}_{v}\right) v \notin \Phi$. Let $S$ denote the unit sphere in $\mathbf{R}^{k}$ centered at the origin. Take any $\mathbf{x} \in S$. Define $\theta_{\mathbf{x}}(t)=\Theta\left(t \mathbf{x}+\mathbf{t}_{v}\right)$ for all $t \in \mathbf{R}$. Thus $\theta_{\mathbf{x}} \in \mathscr{P}_{l}(G)$. Define

$$
\begin{aligned}
& \Psi_{\mathbf{x}}(v)=\left\{t \in[0,1]: \theta_{\mathbf{x}}(t) v \in \Psi\right\}, \\
& \Phi_{\mathbf{x}}(v)=\left\{t \in[0,1]: \theta_{\mathbf{x}}(t) v \in \Phi\right\}, \\
& \Psi_{\mathbf{x}}^{*}(v)=\left\{t \in \Psi_{\mathbf{x}}(v): \theta_{\mathbf{x}}(t) x \in Z_{1}\right\}, \\
& \Phi_{\mathbf{x}}^{\prime}(v)=\bigcup\left\{(a, b) \subset \Phi_{\mathbf{x}}(v):(a, b) \cap \Psi_{\mathbf{x}}^{*} \neq \varnothing\right\}, \text { and } \\
& \Phi_{\mathbf{x}}^{*}(v)=\left\{t \in \Phi_{\mathbf{x}}^{\prime}(v): \theta_{\mathbf{x}}(t) x \in K_{1} \backslash \Omega_{2}\right\} .
\end{aligned}
$$

Let $I=(a, b)$ be any connected component of $\Phi_{\mathbf{x}}^{\prime}(v)$. Since $0 \notin \Phi_{\mathbf{x}}(v)$, we have $a \notin \Phi_{\mathbf{x}}(v)$. Therefore, by equation (14),

$$
\ell\left(\Psi_{\mathbf{x}}(v) \cap I\right)<\varepsilon /(4 k) \ell(I) .
$$

Since $I \cap \Psi_{\mathbf{x}}^{*}(v) \neq \varnothing$, by equations (10), (11), and (13),

$$
\ell\left(\Phi_{\mathbf{x}}^{*}(v) \cap I\right)>(1-1 /(2 k)) \ell(I) .
$$


Define

$$
\begin{aligned}
& \Psi^{*}(v)=\left\{\mathbf{t} \in B: \Theta(\mathbf{t}) v \in \Psi, \Theta(\mathbf{t}) x \in Z_{1}\right\}=\bigcup_{\mathbf{x} \in S} \Psi_{\mathbf{x}}^{*}(v) \mathbf{x}+\mathbf{t}_{v}, \\
& \Phi^{\prime}(v)=\bigcup_{\mathbf{x} \in S} \Phi_{\mathbf{x}}^{\prime}(v) \mathbf{x}+\mathbf{t}_{v}, \quad \text { and } \\
& \Phi^{*}(v)=\left\{\mathbf{t} \in \Phi^{\prime}(v): \Theta(\mathbf{t}) x \in K_{1} \backslash \Omega_{2}\right\}=\bigcup_{\mathbf{x} \in S} \Phi_{\mathbf{x}}^{*}(v) \mathbf{x}+\mathbf{t}_{v} .
\end{aligned}
$$

Due to equations (16) and (17), we can apply Lemma 3.2 for sets $\Psi^{*}(v)$ in place of $E, \Phi^{*}(v)$ in place of $F$, and $\Phi^{\prime}(v)$ in place of $D$, and the constants $\varepsilon_{1}=\varepsilon /(4 k)$ and $\varepsilon_{2}=1 /(2 k)$. Then

$$
m\left(\Psi^{*}(v)\right) \leqslant(\varepsilon / 2) \cdot m\left(\Phi^{*}(v)\right)
$$

Observe that by our choice of $\Phi$, for any two distinct $v_{1}, v_{2} \in \operatorname{Rep}(x)$,

$$
\Phi^{*}\left(v_{1}\right) \cap \Phi^{*}\left(v_{2}\right)=\varnothing .
$$

Now by equations (13), (15), (20), (21), and (18), we get

$$
\begin{aligned}
m\left(\left\{\mathbf{t} \in B: \Theta(\mathbf{t}) x \in \Omega \cap Z_{1}\right\}\right) & \leqslant m\left(\bigcup_{v \in \operatorname{Rep}(x)} \Psi^{*}(v)\right) \\
& \leqslant \sum_{v \in \operatorname{Rep}(x)} m\left(\Psi^{*}(v)\right) \\
& \leqslant(\varepsilon / 2) \cdot \sum_{v \in \operatorname{Rep}(x)} m\left(\Phi^{*}(v)\right) \\
& \leqslant(\varepsilon / 2) \cdot m(B) .
\end{aligned}
$$

Now suppose that possibility (1) also does not hold. Then equation (12) holds. Possibility (3) follows from equations (12), (13), and (22). This completes the proof.

\section{Proofs of the results stated in the introduction.}

Proof of Theorem 1.1. We shall prove the theorem by induction on $\operatorname{dim} G / \Delta$.

Let $\mu$ be a limiting distribution as in Note 3.1. Let $W$ be the subgroup generated by all the unipotent one-parameter subgroups of $G$ preserving $\mu$. By Proposition 4.1, $\operatorname{dim} W>0$. By Theorem 4.2, there exists $H \in \mathscr{H}$ such that $\mu(\pi(S(H, W)))=$ 0 and $\mu(\pi(N(H, W)))>0$. Let $C_{1} \subset N(H, W) \backslash S(H, W)$ be a compact set such that $\mu\left(\pi\left(C_{1}\right)\right)>\varepsilon$ for some $\varepsilon>0$. Let $\Theta$ be as in the hypothesis; then there exists $l \in \mathbf{N}$ such that $\Theta \in \mathscr{P}_{l}\left(\mathbf{R}^{k}, G\right)$. Since $\Theta\left(\mathbf{R}^{k}\right) \subset G_{1}$ and $G_{1} / \Delta$ admits a finite $G_{1}$-invariant measure, applying Theorem 5.2 to $G_{1}$ in place of $G$, we deduce the following: 
there exists a neighbourhood $\Omega$ of $\pi\left(C_{1}\right)$ such that either (i) $\Theta\left(\mathbf{R}^{k}\right) \Delta \subset g N^{1}(H) \Delta$ for some $g \in G$, or (ii) $\mu_{B_{n}}(\Omega)<\varepsilon$ for all large $n \in \mathbf{N}$. Now, if (ii) holds, then $\mu\left(\pi\left(C_{1}\right)\right) \leqslant \varepsilon$, which is a contradiction. Therefore (i) must hold.

Since $g N^{1}(H) g^{-1} \supset \Delta^{0}$, we have $\Theta\left(\mathbf{R}^{k}\right) \subset g N^{1}(H) g^{-1}$. By Theorem 5.1, the orbit $g N^{1}(H) \Delta$ is closed. Also $g N^{1}(H) g^{-1}$ is an algebraic group. Therefore, by Note 2.1, $G=g N^{1}(H) g^{-1}$; that is, $G=N(H)$. Since $\mu(\pi(N(H, W)))>0$, we have that $W \subset H$ and $G=N(H, W)$. Thus $\mu(N \pi((H, W)))=1$. Now, by Theorem $4.2(2), \mu$ is $H$ invariant.

Put $\Lambda=H \Delta$. Since $H \in \mathscr{H}$ and $N(H)=G$, we have that $\Lambda$ is a closed subgroup of $G$. Consider the $G$-equivariant quotient map $q: G / \Delta \rightarrow G / \Lambda$. Let $q_{*}: \mathscr{P}(G / \Delta) \rightarrow$ $\mathscr{P}(G / \Lambda)$ be the map defined as $q_{*} v(E)=v\left(q^{-1}(E)\right)$ for all Borel sets $E \subset G / \Lambda$ and all $v \in \mathscr{P}(G / \Delta)$. The map $q_{*}$ is continuous. Since $\mu_{B_{n}} \rightarrow \mu$, we have that $q_{*}\left(\mu_{B_{n}}\right) \rightarrow$ $q_{*}(\mu)$. Note that for any $\bar{f} \in \mathrm{C}_{\mathrm{c}}(G / \Lambda)$,

$$
\begin{aligned}
\int_{G / \Lambda} \bar{f} d q_{*}\left(\mu_{B_{n}}\right) & =\int_{G / \Delta} \bar{f} \circ q d \mu_{B_{n}} \\
& =\frac{1}{m\left(B_{n}\right)} \int_{B_{n}} \bar{f}(q \circ \Theta(\mathbf{t}) \Delta) d m(\mathbf{t}) \\
& =\frac{1}{m\left(B_{n}\right)} \int_{B_{n}} \bar{f}(\Theta(\mathbf{t}) \Lambda) d \mathbf{t}
\end{aligned}
$$

Since $\Lambda$ contains a nontrivial unipotent one-parameter subgroup of $G$, due to Note 2.2 we have $\operatorname{dim} \Lambda^{0}>\operatorname{dim} \Delta^{0}$. Thus $\operatorname{dim}(G / \Lambda)<\operatorname{dim}(G / \Delta)$. Using the induction hypothesis, we can assume that Theorem 1.1 is valid for the $\Lambda$ in place of $\Delta$. Hence, due to equation (23), $q_{*}(\mu)$ has the following property: there exists a closed subgroup $F$ of $G$ containing $H$ such that $\Theta\left(\mathbf{R}^{k}\right) \subset F, q_{*}(\mu)$ is $F$-invariant, the orbit $F \Lambda$ is closed, and $q_{*}(\mu)(F \Lambda)=1$. Now, since the fibres of $q$ are closed $H$-orbits and $\mu$ is $H$-invariant, we have that $\mu$ is $F$-invariant (cf. [D1, Proposition 1.6]). Since $F \supset H$, we have that $\mu(F \Delta)=q_{*} \mu(F \Lambda)=1$. Let $L$ be any closed subgroup of $G$ containing $\Theta\left(\mathbf{R}^{k}\right)$ such that the orbit $L \Delta$ is closed. Then $\mu(L \Delta)=1$. Hence $F \subset L \Delta^{0}$. Thus in view of Note 3.1 the proof is complete.

Proof of Corollary 1.1. Let $\psi: \mathbf{R}_{+} \rightarrow G$ be the function defined as $\psi(t)=\phi\left(t^{1 / k}\right)$ for all $t \geqslant 0$. Now argue just as in the proof of Theorem 1.1, replacing $\Theta$ by $\psi$. There are exactly three places where we use that $\Theta$ is a regular algebraic function: (1) Theorem 3.1, (2) Proposition 4.1, and (3) Proposition 5.3. Therefore, if the corresponding statements are shown to hold for $\psi$ in place of $\Theta$, we would get a proof of the corollary.

First we have Lemma 4.2, which replaces $\theta$ by $\psi$ in Lemma 4.1. Therefore, in the proof of Proposition 4.1, we can use Lemma 4.2 in place of Lemma 4.1 and obtain the same conclusion for $\psi$ in place of $\Theta$. Also, due to Lemma 3.1, it is clear that Theorem 3.1 and Proposition 5.3 are valid for $\psi$ in place of $\Theta$ or $\theta$. This completes the proof. 
Proof of Corollary 1.2. Let $S$ denote the unit sphere in $\mathbf{R}^{k}$ and $\sigma$ the rotation invariant probability measure on $S$. Using polar decomposition, for any $f \in$ $\mathrm{C}_{\mathrm{c}}(G / \Delta)$ and any $T>0$,

$$
\frac{1}{m\left(B_{T}\right)} \int_{\mathbf{t} \in B_{T}} f(\Theta(\mathbf{t}) \Delta) d m(\mathbf{t})=\int_{\mathbf{x} \in S} d \sigma(\mathbf{x})\left(\frac{1}{T^{k}} \int_{0}^{T^{k}} f\left(\Theta\left(t^{1 / k} \mathbf{x}\right) \Delta\right) d t\right) .
$$

For every $\mathbf{x} \in S$, define $\theta_{\mathbf{x}}(t)=\Theta(t \mathbf{x})$ for all $t \in \mathbf{R}$. Let $F_{\mathbf{x}}$ be the smallest closed subgroup $L$ of $G$ such that $L \supset \Delta^{0}, L \supset \theta_{\mathbf{x}}(\mathbf{R})$, and the orbit $L \Delta$ is closed.

Due to Corollary 1.1 , for any $f \in \mathrm{C}_{\mathrm{c}}(G / \Delta)$,

$$
\lim _{T \rightarrow \infty} \frac{1}{T} \int_{0}^{T} f\left(\theta_{\mathbf{x}}\left(t^{1 / k}\right) \Delta\right) d t=\int_{F_{\mathbf{x}} \Delta} f d \mu_{\mathbf{x}}
$$

where $\mu_{\mathbf{x}}$ denotes the unique $F_{\mathbf{x}}$-invariant probability measure supported on the closed orbit $F_{\mathbf{x}} \Delta$.

By Proposition 2.1, we have $F_{\mathbf{x}} \in \mathscr{H}$. The set $\Theta^{-1}\left(F_{\mathbf{x}}\right) \cap S$ is an analytic submanifold of $S$. Hence, if $\operatorname{dim}\left(\Theta^{-1}\left(F_{\mathbf{x}}\right) \cap S\right)=\operatorname{dim} S$, then $F_{\mathbf{x}} \supset \Theta\left(\mathbf{R}^{k}\right)$. Note that if $\mathbf{y} \in \Theta^{-1}\left(F_{\mathbf{x}}\right) \cap S$, then $F_{\mathbf{y}} \subset F_{\mathbf{x}}$. Put

$$
E=\left\{\mathbf{x} \in S: \operatorname{dim}\left(\Theta^{-1}\left(F_{\mathbf{x}}\right) \cap S\right)<\operatorname{dim}(S)\right\}
$$

Now $\sigma\left(\Theta^{-1}\left(F_{\mathbf{x}}\right) \cap S\right)=0$ for every $\mathbf{x} \in E$. Since $\mathscr{H}$ is a countable collection, we have that

$$
E=\bigcup_{\mathbf{x} \in E}\left(\Theta^{-1}\left(F_{\mathbf{x}}\right) \cap S\right)
$$

is a countable union. Therefore

$$
\sigma(E)=0
$$

Now let $F$ denote the smallest closed subgroup $L$ of $G$ such that $L \supset \Delta^{0}, L \supset$ $\Theta\left(\mathbf{R}^{k}\right)$, and the orbit $L \Delta$ is closed. Then $F_{\mathbf{x}}=F$ for all $\mathbf{x} \in S \backslash E$. Let $\mu_{F}$ denote the unique $F$-invariant probability measure on $F \Delta$.

Let $\varepsilon>0$ and $f \in \mathrm{C}_{\mathrm{c}}(G / \Delta)$ be given. For any $R>0$, the set $W_{R}$, consisting of all $\mathbf{x} \in S$ such that

$$
\left|\frac{1}{T^{k}} \int_{0}^{T^{k}} f\left(\theta_{\mathbf{x}}\left(t^{1 / k}\right) \Delta\right) d t-\int_{F \Delta} f d \mu_{F}\right|<\varepsilon / 2
$$

for all $T \geqslant R$, is Borel measurable. Also note that $W_{R_{1}} \subset W_{R_{2}}$ for all $R_{1} \leqslant R_{2}$. 
Now, due to equation (25), we have

$$
S \backslash E=\bigcup_{R>0} W_{R}
$$

Therefore, by equation (26), there exists $R>0$ such that

$$
\sigma\left(S \backslash W_{R}\right)<\frac{\varepsilon}{(4 \cdot \sup |f|)}
$$

Now, by equations (24), (27), and (28), for every $T \geqslant R$, we get

$$
\begin{aligned}
& \left|\frac{1}{B_{T}} \int_{\mathbf{t} \in B_{T}} f(\Theta(\mathbf{t}) \Delta) d \mathbf{t}-\int_{F \Delta} f d \mu_{F}\right| \\
& \quad \leqslant \int_{\mathbf{x} \in W_{R} \cup\left(S \backslash W_{R}\right)}\left|\frac{1}{T^{k}} \int_{0}^{T^{k}} f\left(\theta_{\mathbf{x}}\left(t^{1 / k} \mathbf{x}\right) \Delta\right) d t-\int_{F \Delta} f d \mu_{F}\right| d \sigma(\mathbf{x}) \\
& \quad<\varepsilon .
\end{aligned}
$$

This completes the proof.

Proof of Corollary 1.3. For $i=1, \ldots, k$, define $\theta_{i}(t)=\exp \left(t b_{i}\right)$ for all $t \in \mathbf{R}$. Define $\Theta\left(t_{1}, \ldots, t_{k}\right)=\theta_{k}\left(t_{k}\right) \cdots \theta_{1}\left(t_{1}\right)$ for all $\left(t_{1}, \ldots, t_{k}\right) \in \mathbf{R}^{k}$.

Note that due to [R1, Lemma 1.4], the Lebesgue measure on $\mathbf{R}^{k}$ projects under $\Theta$ to a Haar measure on $N$. Note that if $G$ a real algebraic group and $N$ is an algebraic unipotent subgroup, we have that $\theta_{i}$ is a regular algebraic map for each $i=1, \ldots, k$. And the corollary immediately follows from Theorem 1.1.

Now in the general case we argue just as in the proof of Theorem 1.1. First note that $\Theta \in \mathscr{P}_{l}\left(\mathbf{R}^{k}, G\right)$ for $l=\operatorname{dim} G-1$. Therefore Theorem 3.1 and Proposition 5.3 are applicable to $\Theta$. Since each $\theta_{i}$ is a nontrivial unipotent one-parameter subgroup of $G$, Proposition 4.1 holds in this case. Now, in view of the remarks made in the proof of Corollary 1.1, the proof of Theorem 1.1 yields the validity of the corollary.

\section{REFERENCES}

[CFS] I. P. CoRnfeld, S. V. Fomin, AND YA. G. Sinal, Ergodic Theory, Springer-Verlag, New York, 1982.

[D1] S. G. DANI, Invariant measures of horospherical flows on noncompact homogeneous spaces, Invent. Math. 47 (1978), 101-138.

[D2] - A simple proof of Borel's density theorem, Math. Z. 174 (1980), 81-94.

[DM1] S. G. DANi AND G. A. MARGUlis, Values of quadratic forms at primitive integral points, Invent. Math. 98 (1989), 405-424.

[DM2] - Orbit closures of generic unipotent flows on homogeneous spaces of SL(3, R), Math. Ann. 286 (1990), 101-128. 
[DM3] - "Limit distributions of orbits of unipotent flows and values of quadratic forms" in I. M. Gelfand Seminar Adv. Soviet Math. 16, Amer. Math. Soc., Providence, 1993, 91-137.

[DS] S. G. Dani AND J. Smillie, Uniform distribution of horocycle orbits for Fuchsian groups, Duke Math. J. 51 (1984), 185-194.

[MS] S. MOZES AND N. A. SHAH, On the space of ergodic invariant measures of unipotent flows, to appear in Ergodic Theory Dynamical Systems.

[R1] M. RATNER, Strict measure rigidity for unipotent subgroups of solvable groups, Invent. Math. 101 (1990), 449-482.

[R2] - On measure rigidity of unipotent subgroups of semisimple Lie groups, Acta Math. 165 (1990), 229-309.

[R3] - On Raghunathan's measure conjecture, Ann. of Math. (2) 134 (1991), 545-607.

[R4] Raghunathan's topological conjecture and distributions of unipotent flows, Duke Math. J. 63 (1991), 235-280.

[R5] - Invariant measures and orbit closures for unipotent actions on homogeneous spaces, Geom. Funct. Anal. 4 (1994), 236-256.

[S] N. A. SHAH, Uniformly distributed orbits of certain flows on homogeneous spaces, Math. Ann. 289 (1991), 315-334.

School of Mathematics, Tata Institute of Fundamental Research, Homi Bhabha Road, BOMBAY 400 005, INDIA; nimish@tifrvax.tifr.res.in 